\title{
Management of Obstetric Emergencies in a Tertiary Hospital in Cameroon: A Milestone for End of Preventable Maternal Deaths
} \author{
Diane Estelle Kamdem1, Moustapha Bilkissou1, Félix Adolphe Elong2,6, \\ Dominique Djomo Tamchom, Julie Ngo Batta1, Emile T. Mboudou ${ }^{1,7}$ \\ ${ }^{1}$ Douala Gynaeco-Obstetric and Paediatric Hospital, Douala, Cameroon \\ ${ }^{2}$ Faculty of Health Sciences, University Buea, Buea, Cameroon \\ ${ }^{3}$ Douala General Hospital, Douala, Cameroon \\ ${ }^{4}$ Faculty of Medicine and Biomedical Sciences of Ngaoundere, Garoua, Cameroon \\ ${ }^{5}$ Regional Hospital Limbe, Limbe, Cameroon \\ ${ }^{6}$ Regional Hospital Buea, Buea, Cameroon \\ ${ }^{7}$ Faculty of Medicine and Biomedical Sciences, University of Yaounde I, Yaounde, Cameroon \\ Email: *rtchounzou@yahoo.fr
}

Robert Tchounzou ${ }^{1,2^{*}}$, Theophile Nana Njamen ${ }^{2,3}$, Alphonse Nyong Ngalame ${ }^{1}$, Vanessa Baleba ${ }^{1}$, Inna Rakya ${ }^{1,4}$, Darolles Mwadjie Wekam1, André Gaetan Simo Wambo ${ }^{2,5}$, Humphry Neng Tatah',

How to cite this paper: Tchounzou, R., Njamen, T.N., Ngalame, A.N., Baleba, V., Rakya, I., Wekam, D.M., Wambo, A.G.S., Tatah, H.N., Kamdem, D.E., Bilkissou, M., Elong, F.A., Tamchom, D.D., Batta, J.N. and Mboudou, E.T. (2020) Management of Obstetric Emergencies in a Tertiary Hospital in Cameroon: A Milestone for End of Preventable Maternal Deaths. Open Journal of $\mathrm{Ob}$ stetrics and Gynecology, 10, 1749-1762. https://doi.org/10.4236/ojog.2020.10120158

Received: October 31, 2020

Accepted: December 27, 2020

Published: December 30, 2020

Copyright $\odot 2020$ by author(s) and Scientific Research Publishing Inc. This work is licensed under the Creative Commons Attribution International License (CC BY 4.0).

http://creativecommons.org/licenses/by/4.0/ (c) (i) Open Access

\begin{abstract}
Background: Maternal mortality was insufficiently reduced in Cameroon in 2015 despite the adoption of Millennium development goals. To tackle the situation and meet the sustainable Millennium goals target of 140/100,000 live births by 2030, the Government adopted the strategies of building reference hospitals where high quality obstetric care, timely and optimal management of obstetric emergencies will be offered. The objective of this study was to describe the patterns of obstetric emergencies in Douala Gynaeco-obstetric and Paediatric Hospital, evaluate the outcomes of their management and the contribution to maternal mortality. Patients and Methods: 418 patients with obstetric emergencies were included in a two-phase cross-sectional study. Data were retrieved from patients' case notes during the retrospective phase and a questionnaire filled for each case received during the prospective phase. Patterns of obstetric emergencies were determined and for each, the following were analysed: patient managed in this hospital or referred from other hospitals, management according to hospital guidelines, timing of care, result of management (recovery with no admission in ICU (intensive care unit), admission in ICU, death). Factors associated with each case of death were analysed. Results: The patterns of obstetric emergencies $(O E)$ were dominated by HDP (hypertensive diseases in pregnancy) (20.57\%), abortions (14.83\%),
\end{abstract}


Ectopic pregnancies (13.87\%), Acute foetal distress (13.15\%) and Obstructed labour (9.56\%). PPH (post partum haemorrhage) represented $7.65 \%$ and Sikcle cell crisis (SCA) $0.91 \%$. $40 \%$ of cases were referred from other hospitals. Six cases of deaths were recorded with a global case fatality of $1.43 \%$. The causes of death were PPH, HDP, and Sickle cell anaemia 33.33\% each. The case fatality of SCA was $50 \%$, disclosing our worst performance. Conclusion: Management of OE following standardized hospital guidelines, reinforcement of referral systems, upgrading obstetrical services with ICU will result in least adverse maternal outcomes and especially reduced maternal mortality.

\section{Keywords}

Obstetric Emergencies, Tertiary Hospital, Maternal Outcome, Maternal Death

\section{Introduction}

Reducing maternal mortality by the year 2015 was one of the most challenging millennium goals pledged by the United Nations' members in 2000. The target was to reduce by $75 \%$ the high maternal mortality ratios of the 1990 's by the year 2015 [1] [2]. There has been a period of escalation of maternal mortality in low and middle income countries (LMIC) and especially in sub-Saharan Africa where the majority of the world maternal death was recorded [1] [3] [4] [5]. The evaluation of the achievement of the goal showed a global reduction of $44 \%$ worldwide but with a lot of disparities in the efforts of countries [2] [6] [7]. In a new publication of WHO, entitled Ending preventable maternal mortality (EPMM), new target at country level was established at a maternal mortality rate (MMR) not more than 140/100,000 live births [8]. In Cameroon MMR has almost doubled from 430/100,000 live births in 1998 to 782 in 2011 [3] [5] [9]. To respond to this situation, the Cameroon government has adopted amongst other strategies the one of constructing tertiary hospitals with high technology equipment, skilled health workers and other facilities with the missions of offering high quality obstetric care (state of the art obstetric care). These hospitals are expected to offer evidence based reproductive health care to women to minimise maternal deaths. Tebeu et al. in a study conducted in a tertiary hospital in Cameroon, in 2010, reported an MMR of 287.5\%/100,000 live births as compared to 782 at National level [9]. Amongst the strategies proven efficient to reduce maternal and perinatal deaths are management of obstetric and neonatal emergencies, the antenatal care, skilled birth attendants, and good health care delivery system. Appropriate and timely management of obstetric emergencies in hospital (reducing the third delay) has been proven as an efficient strategy to reduce maternal and neonatal mortality by many authors [8] [10]. In a study conducted in Nigeria, Lamina Mustafa et al. [11] found that obstetric emergencies constitute $18.5 \%$ of deliveries and represented $70.6 \%$ of causes of maternal death. Di- 
rect obstetric causes of $\mathrm{MM}$ in Cameroon include post partum haemorrhage (29.2\%), unsafe abortions (25\%), ectopic pregnancy (12.5\%), hypertensive diseases in pregnancy $(8.3 \%)$ and indirect causes include malaria $(8.2 \%)$ and cardiac diseases in $4.2 \%$ [9]. Studies designed to determine the case fatality of individual causes of maternal and perinatal death are scarce in Cameroon. The objective of this study was to describe the patterns of obstetric emergencies in HGOPED, the outcome of management and the contribution of each of these to maternal death.

\section{Patients and Methods}

\subsection{Study Site}

DGOPH is a tertiary Hospital created in 2013 and inaugurated in 2015, mainly dedicated to mother and child care. The three missions of this institution comprise of 1) providing high standard and quality care to targeted patients, 2) contributing to Medical training and 3) healthcare related research. The infrastructure and equipment are of high quality; human resources include 9 obstetrician and Gynaecologists, 5 Paediatricians, 3 intensive care workers, radiologists, surgeons, qualified midwives, nurses and many other categories of workers involved in management of emergencies. The laboratory service host one of the most functional blood bank units of the country where blood products like whole blood, frozen plasma, packed cells, platelets are always available.

In this study, an obstetrical emergency was any life-threatening medical condition during pregnancy, labour or post partum. The scope included direct obstetrical causes and acute complications of some indirect causes like malaria, cardiac diseases, and sickle cell anaemia.

\subsection{Work Organization and Management of Obstetrical Emergencies (OE) at DGOPH}

The work organization in the department of Obstetrics and Gynaecology is divided into two shifts for medical physicians, residents in Obstetrics and Gynaecology: the daily routine activities and the call duties. Besides routine duties, the service is covered every day by a team comprised of the above mentioned workers' categories, supervised by a consultant obstetrician and gynaecologist who work in close collaboration with the team of midwives, nurses and other paramedical staff. The hospital has elaborated guidelines for the standardized management of the most frequent $\mathrm{OE}$ and gynaecological emergencies. Thought not exhaustive, these guidelines are qualitatively and quantitatively updated on regular basis.

\subsection{Study Design}

We carried out a two phases cross sectional study with the retrospective phase consisting of review of service delivery records of cases of obstetrical emergencies from August 2015 to December 2017. In the prospective phase same infor- 
mation was recorded to refine the analysis from January 2018 to December 2019.

\subsection{Inclusion Criteria}

All cases of obstetrical emergencies received and managed during the period of study were included.

\subsection{Exclusion Criteria}

Those with incomplete data in the files (retrospective phase) or who died less than two hours after admission in our maternity (prospective phase) were excluded. We also excluded all patients who refused to participate in the study.

\subsection{Variables Collected and Analyzed}

We compiled the following information from patients' files or directly from patients or relatives in the prospective phase and entered in a pre-tested data collection sheet: 1) Socio-demographic data such as age, level of education and profession; 2) obstetric information: parity, gestational age and 3) therapeutic information: patient managed in this hospital or referred, diagnosis or type of obstetric emergency, management according to hospital guidelines or no, obstetrician present at arrival or not, maternal outcome (successful with no admission in intensive care unit (ICU), admission in ICU, death, referred to other health facilities).

Case fatality was determined for each type of obstetric emergency and in case of death, factors involved were analysed (existence of protocol of management, timing of management, availability of carers, and availability of blood products) to evaluate the use of evidence-based and standard health care procedures.

Data analysis was done using SPSS version 23 software.

\subsection{Ethical Considerations}

We obtained clearance from the hospital institutional review board and the General Manager granted administrative authorization.

\section{Results}

The results were similar for the retrospective and the prospective phases and are analysed conjointly.

\subsection{Socio-Demographic Characteristics of Study Population}

Out of the 2634 deliveries recorded during the study period, 418 (15.86\%) presented as obstetrical emergencies. Table 1 summarises the socio-demographic characteristics of the study population. The mean age of patients was $29.37 \pm$ 5.76 years with extreme ages of 16 and 47 years. 284 (67.95\%) of study population had university level of education and only 29 (6.94\%) primary level. The majority of our participants had a revenue generating profession with $20.33 \%$ of them being civil servants, $20.33 \%$ self-employed and $21.29 \%$ workers of the private sector. 
Table 1. Socio-demographic distribution of patients.

\begin{tabular}{|c|c|c|}
\hline \multicolumn{3}{|c|}{ Frequency Percentage (\%) } \\
\hline \multicolumn{3}{|c|}{ Profession $(n=418)$} \\
\hline Unemployed & 52 & 12.45 \\
\hline Self employed & 85 & 20.33 \\
\hline Civil servant & 85 & 20.33 \\
\hline Private salary & 89 & 21.29 \\
\hline Students & 107 & 25.60 \\
\hline \multicolumn{3}{|c|}{ Parity $(\mathrm{n}=418)$} \\
\hline $0-1$ & 206 & 49.28 \\
\hline $2-3$ & 108 & 25.83 \\
\hline $4-5$ & 68 & 16.26 \\
\hline$>5$ & 36 & 8.62 \\
\hline Total & 418 & \\
\hline \multicolumn{3}{|c|}{ Level of education $(n=418)$} \\
\hline Primary & 29 & 6.94 \\
\hline Secondary & 105 & 25.11 \\
\hline University & 284 & 67.95 \\
\hline Total & 418 & 100 \\
\hline
\end{tabular}

\subsection{Primary Site of Patients' Management}

Forty per cent of patients with obstetric emergencies (167) were referred from other health facilities while $251(60 \%)$ started management in the study site.

Table 2 highlights the patterns of obstetric emergencies recorded during the study period. Hypertensive diseases in pregnancy (HDP) mostly Preeclampsia/eclampsia (PEE) constituted the most frequently encountered 86/418 (20.57\%) followed by ectopic pregnancy, 58 (13.87\%).

\subsection{Maternal Outcome after Management}

Out the 418 patients, $412(98.56 \%)$ were managed successfully with only $19.61 \%$ admitted in ICU. Six (6) maternal deaths were recorded giving a case fatality of 6/418 (1.4\%) of obstetric emergencies and an MMR of 228/100,000 live births. The causes of death are summarized on Table 3.

Of the 418 patients, 336 (80.38\%) were managed without admission in ICU. It must be noted that some of the patients who needed intensive care were managed in the routine wards because of limited places and equipment in ICU.

Three diseases namely post partum haemorrhage (PPH), sickle cell anaemia (SCA), and preeclampsia/eclampsia (PEE) dominated the causes of maternal death with equal proportion of $2(33.33 \%)$ each. The case fatality for PPH was $2 / 32$ (6.25\%), $2 / 4$ (50\%) for SCA and $2 / 86(2.32 \%)$ for PEE. 
Table 2. Patterns of obstetric emergencies.

\begin{tabular}{ccc}
\hline Obstetrical emergency & Number & Percentage (\%) \\
\hline Hypertensive diseases in pregnancy (HDP) & 86 & 20.57 \\
Post partum haemorrhage (PPH) & 32 & 7.65 \\
Placenta previa (PP) & 16 & 3.82 \\
Placenta abruption (AP) & 13 & 3.11 \\
Abortions (AB) & 62 & 14.83 \\
Ectopic pregnancy (EP) & 58 & 13.87 \\
Obstructed labour (OL) & 40 & 9.56 \\
Sickle cell disease crisis (SCA) & 4 & 0.91 \\
Acute foetal distress (AFD) & 55 & 13.15 \\
Other indirect causes (OIC) & 52 & 12.44 \\
Total & 418 & 100 \\
\hline
\end{tabular}

Table 3. Therapeutic characteristics and maternal outcomes of OE.

\begin{tabular}{cccccccc}
\hline OE & Number & Percent $(\%)$ & NICU & ICU & Ref & MIS & DEATH \\
\hline HDP & 86 & 20.57 & 26 & 60 & 42 & 44 & 2 \\
PPH & 32 & 7.65 & 26 & 6 & 17 & 15 & 2 \\
PP & 16 & 3.82 & 16 & 0 & 4 & 12 & 0 \\
AP & 13 & 3.11 & 11 & 2 & 3 & 10 & 0 \\
AB & 62 & 14.83 & 62 & 0 & 7 & 55 & 0 \\
EP & 58 & 13.87 & 58 & 0 & 18 & 40 & 0 \\
OL & 40 & 9.56 & 40 & 0 & 13 & 27 & 0 \\
SCA & 4 & 0.91 & 0 & 4 & 4 & 0 & 2 \\
AFD & 55 & 13.15 & 55 & 0 & 27 & 28 & 0 \\
OIC & 52 & 12.44 & 42 & 10 & 32 & 20 & 0 \\
Total & 418 & 100 & 336 & 82 & 167 & 251 & 6 \\
\hline
\end{tabular}

Legend: NCIU: not admitted in intensive care unit; ICU: admitted in intensive care unit; Ref: referred from other facilities; MIS: started management in site.

\subsection{Management Processing Time}

As shown in Table 4, the minimal processing time was 10 minutes and the maximum 1140. The shorted mean processing time was recorded for abortions and the longest for sickle cell anaemia. 
Table 4. Mean and median management processing time.

\begin{tabular}{ccccc}
\hline \multirow{2}{*}{ Obstetrical emerg } & \multicolumn{2}{c}{ Processing time } & & \\
\cline { 2 - 3 } & Min & Max & & \\
Global management & 10 & 1140 & $65.46 \pm 99.7$ & $45(35-60)$ \\
PPH & 20 & 180 & $35.15 \pm 42.5$ & $20(15-30)$ \\
EP & 22 & 180 & $45.40 \pm 28.74$ & $40(37-45)$ \\
RPH & 29 & 48 & $39.29 \pm 6.26$ & $41(35-45)$ \\
PP & 30 & 120 & $62 \pm 31.90$ & $51(42.25-75)$ \\
HDP & 15 & 180 & $61.53 \pm 85.49$ & $40(25-62)$ \\
Abortions & 15 & 130 & $32.65 \pm 25$ & $27.50(23-63)$ \\
SCA & 20 & 1140 & $105.29 \pm 222.61$ & $50(45-60)$ \\
Labour dystocia & 25 & 360 & $86.69 \pm 83.15$ & $50(45-112.50)$ \\
Other indirect causes & 10 & 1140 & $104.29 \pm 222$ & $50(45-60)$ \\
\hline
\end{tabular}

Min: minimal; Max: maximal.

\section{Discussion}

Our study has shown a global obstetric emergency rate of 418/2634 (15.86\%) which is consistent with the figures of Mustapha Lamina et al. who found a rate of $18.5 \%$ in Nigeria [11]. We recorded 6 maternal deaths during the period under study, which represented an MMR of 228/100,000 live births slightly different from the figures found by Fomulu et al. in 2013 in a retrospective study in a reference hospital in Yaounde between 2002 and 2006 $(365 / 100,000)$ and Tebeu et al. in 2015 in the Yaoundé Gynaeco-obstetric and Paediatric hospital $(287.5 / 100,000)$ [3] [9]. Our relative low rate may be explained by the smallest sample size. Like the previous authors who evaluated the MMR in the reference hospitals of the country between 2000 and 2015, this rate was lower than the national level which was 782/100,000 and other rates reported in some reference hospitals in sub-Saharan Africa [12] [13]. Lower rates are recorded in Asian countries of the same levels of care, 141/100,000 live births in India $(n=104 / 73,935)$ [14]. All the cases of maternal death were due to obstetric emergencies with $66.66 \%(n=4)$ due to direct causes and $33.33 \%(n=2)$ to indirect causes. It is well established that obstetric emergencies constitute the major causes of maternal deaths in LMIC. Table 5 summarises pictures of maternal deaths reported in some LMIC.

Patterns of obstetric emergencies were dominated by hypertensive diseases in pregnancy, EP, PPH and obstructed labour which is not different from what is described in other low or middle income settings [1] [5] [9] [11]. 
Table 5. Picture of maternal reported by some authors in LMIC.

\begin{tabular}{|c|c|c|c|c|}
\hline & MMR & GOE & Direct causes & Indirect causes \\
\hline Our results & $228(n=6)$ & $418 / 2634$ & $100 \%$ & 0 \\
\hline Fomulu et al. (2002-2006) [3] & $365(n=39 / 10662)$ & - & $89.7 \%$ & $10.3 \%$ \\
\hline Tebeu PM et al. (2006-2010) [9] & $287.5(n=26 / 9045)$ & - & $75 \%$ & $25 \%$ \\
\hline Mustapha Lamina et al. (2005-2007) [11] & - & $262 / 1420$ & $70.6 \%$ & $29.4 \%$ \\
\hline Papa Dasri et al. (2008-2012) [14] & 141 & $106 / 73,935$ & $90 \%$ & $10 \%$ \\
\hline
\end{tabular}

Post partum haemorrhage (PPH) represented one of the most frequent causes of maternal death in our study (7.65\%). Two out 32 patients who presented with PPH died, making a case fatality of $6.25 \%$. Case fatality of PPH reported in LMIC varies widely (1.3\% to $27.3 \%$ ) with countries and levels of care and also when evidence-based interventions are implemented or not [15]-[20]. Ezugwu EC et al. reported that case fatality of PPH without evidence-based health intervention will be as high as $13.6 \%$ as compared to $2.5 \%$ when timely and standardized management is applied [16]. Determinants of death related to post partum haemorrhage include women's characteristics, pre-delivery maternal anaemia, lack of blood products, unskilled birth attendants, inadequate or no antenatal care, transfer from other health facilities, delays in management in the hospital amongst others [15] [18] [19] [20]. All the cases of maternal death recorded in our study were referred from other health facilities with critical conditions which warranted admission in intensive care unit. The role of poor referral system has been demonstrated by other authors in Cameroon like Belinga Etienne et al. [21] who quoted that $70 \%-90 \%$ of maternal deaths are due to referred cases according to Perrin in Benin.

Hypertensive diseases in pregnancy (HDP) (mostly preeclampsia/eclampsia) constituted $33.33 \%$ of maternal deaths (MD). According to WHO, HDP is one of the four main causes of maternal death (WHO report 2019) and Lale Say et al. reporting a global maternal death causes according to WHO review found these to represent $11.6 \%$ to $21 \%$ causes of MD [10]. In studies conducted in tertiary hospitals in Cameroon, Tebeu and coll. And Fomulu et al. 8.3\% and 15.4\% respectively [3] [9] while Dasari et al. in India reported HDP to represent $17 \%$ of causes of MD [14]. The case fatality of HDP was $2.32 \%$ which is similar to the outcome of few authors in sub-Saharan Africa like Edward T. Dassah et al. in Ghana (2.2\%) and EC. Ezugwu in Nigeria (2.5\%) [16] [22] but in constrats was very low compared to $11 \%$ described by Rob Mooij et al. in Tanzania [23]. Mekoya D. Mengistu, TilatumRuma and coll. reported no death in a retrospective study enrolling 156 cases of HDP in Addis Ababa. We attribute our relative good performance to many factors including:

1) The existence of management protocols in the service to which almost all carers are strictly bound; 
2) The availability of skilled workers (obstetricians, midwife, and reanimators) when needed;

3) The availability of drugs (antihypertensive drugs, magnesium sulphate);

4) The Presence of an ICU where critical cases needing special attention are admitted though with insufficient beds and resuscitation equipment. In fact, our protocol of management recommends, in accordance with the state of the art in developed countries that all cases of severe preeclampsia and eclampsia be admitted in ICU and hospitals with obstetric units should have an obstetric ICU [24] but we could not achieve this objective because of the aforementioned reasons. This is a shortcoming in the management of emergencies in general and HDP in particular.

5) Concerning the timing of management, the mean proceeding time was $61.53 \pm 85.49$ (see Table 4) with extremes of 15 minutes to 3 hours. American college of Obstetricians and Gynecologists (ACOG) recommends that expeditious management of confirmed severe HDP should be initiated within $30-60$ minutes [25]. Our proceeding time did not always fall within this timeline and delays of up to 3 hours were observed due to several factors including frequent stoke outs of emergency drugs, lack of financial means (payment was made out of patients pockets!) and at time absence of one or many members of the multidisciplinary therapeutic team.

We admitted 4 cases of sickle cell anaemia (SCA) in pregnancy; all referred from other peripheral hospitals with acute onset complications. The reasons for referral included severe anaemia, pains crisis or the two complications combined. All the 4 cases were admitted in ICU during part or all the duration of their hospital stay. The 4 patients delivered through caesarean section and 2 (50\%) died in the early post caesarean period in severe acute respiratory syndrome which we suspected to be due to acute thoracic syndrome. The case fatality of this obstetric emergency appears extremely high compared to what is reported in the literature that ranges from almost no maternal mortality in United Kingdom and USA [26] [27], 0.4\% (total of 255 patients) in Saudi Arabia [28], 5.98 relative risk of death in a systemic review conducted by Eugene Oteng Ntim et al. in 2014 [29] to $11.4 \%$ in a study by Projestine S. Muganyizi et al. in Tanzania [30]. This high mortality can be explained by the reduced number of cases, the fact that patients were all referred in critical conditions but also can delineate the suboptimal performance of our ICU which is not specialized in such diseases.

The other diseases were successfully managed with some mild adverse maternal outcomes but no maternal death. Contrary to other studies carried in Cameroon and other sub-Saharan countries which displayed an important contribution of abortions (up to 25\%) and sepsis, Ectopic pregnancy to maternal death, we recorded no death for these conditions. Although we attribute the relative good performance to the limited number of our sample size, the role of enough qualified staff, the relatively good plateau technique, the availability of management guidelines appear to be contributing factors. 


\section{Conclusion}

Obstetric emergencies were high in the DGOPH, representing $15.4 \%$ of all live births. The management of these emergencies resulted in a relatively low rate of adverse maternal outcomes and only 6/418 deaths were recorded making a case fatality of $1.43 \%$. Of the causes of maternal death, the case fatality of PPH and HDP was comparable to the high performance hospitals in LMIC. Determinants of this performance include the plateau technique, the quality of personnel, the existence of treatment guidelines and an ICU. These findings suggest that the strategy of building reference hospital where "state of the art obstetric care" can be implemented is an important milestone in the attainment of the sustainable millennium goals.

\section{Authors Contribution}

Robert Tchounzou, Theophile Nana Njamen Vanessa Baleba, Alphonse Ngalame Nyong, Inna Rakya, Humphry Neng Tatah designed the study and wrote the protocol; Moustapha Bilkissou, Diane Estelle Kamdem, Julie Ngo Batta and Darolles Mwadjie Wekam participated in patients recruitment. Robert Tchounzou wrote the manuscript, André Gaetan Simo Wambo, Theophile Nana Njamen, Dominique Djomo Tamchom revised the manuscript. Emile TMboudou supervised the manuscript writing. All the authors approved the final version.

\section{Acknowledgements}

We are immensely grateful to all the medical and technical staff of the Gynecology and Obstetrics Service, the emergency unit, the intensive care unit, the imaging and laboratory units, the theatre and the outpatient department of Gynecology and Obstetrics and to the general administration of the DGOPH for their contribution in patients' management and in keeping the records. Special thanks go to Drs. Noura Benmoussa, Eyanga Olivia and Mrs. Chuo Yvette.

\section{Conflicts of Interest}

The authors declare no conflicts of interest regarding the publication of this paper.

\section{References}

[1] World Health Organization, UNICEF, United Nations, Department of Economic and Social Affairs, Population Division, World Bank Trends in Maternal Mortality: 1990 to 2015. Estimates by WHO, UNICEF, UNFPA, World Bank Group and the United Nations Population Division.

[2] Bryce, J., Black, R.E. and Victora, C.G. (2013) Millennium Development Goals 4 and 5: Progress and Challenges. BMC Medicine, 11, 225.

https://doi.org/10.1186/1741-7015-11-225

[3] Fomulu, F.J., Tchana, T.M., Nana, N.P., Mbu, R. and Kasia, J.M. (2013) Mortalité maternelle à l'hôpital général de Yaoundé: Etude rétrospective sur 5 années (2002-2006). Health Sciences and Disease, 10, 5-10. 
[4] Agan, T., Archibong, E., Ekabua, J., Ekanem, E., Abeshi, S.E., Edentekhe, T., et al. (2010) Trends in Maternal Mortality at the University of Calabar Teaching Hospital, Nigeria, 1999-2009. International Journal of Women's Health, 2, 249-254. https://doi.org/10.2147/IJWH.S11971

[5] Wirsiy, F.S., Ako-Arrey, D.E., Njukeng, P.A., Manjong, F.T., Lukong, L.K., Lekedji, M.Y., et al. (2019) Maternal Mortality in Cameroon: A Critical Review of Its Determinants. Journal of Neonatal and Gynaecology, 2, 11.

[6] Maternal Health Task Force (2017) The Sustainable Development Goals and Maternal Mortality.

[7] Kassebaum, N.J., Bertozzi-Villa, A., Coggeshall, M.S., Shackelford, K.A., Steiner, C., Heuton, K.R., et al. (2014) Global, Regional, and National Levels and Causes of Maternal Mortality during 1990-2013: A Systematic Analysis for the Global Burden of Disease Study 2013. The Lancet, 384, 980-1004.

[8] WHO (2020) Strategies toward Ending Preventable Maternal Mortality (EPMM). World Health Organization, Geneva.

[9] Tebeu, P.M., Halle-Ekane, G., Itambi, M., Robinson, E., Yvette, M. and Nelson, F. (2015) Maternal Mortality in Cameroon: A University Teaching Hospital Report. Pan African Medical Journal, 21, 16. https://doi.org/10.11604/pamj.2015.21.16.3912

[10] Say, L., Chou, D., Gemmill, A., Tunçalp, Ö., Moller, A.-B., Daniels, J., et al. (2014) Global Causes of Maternal Death: A WHO Systematic Analysis. The Lancet Global Health, 2, e323-e333. https://doi.org/10.1016/S2214-109X(14)70227-X

[11] Mustafa Adelaja, L. and Olufemi Taiwo, O. (2011) Maternal and Fetal Outcome of Obstetric Emergencies in a Tertiary Health Institution in South-Western Nigeria. ISRN Obstetrics and Gynecology, 2011, Article ID: 160932. https://doi.org/10.5402/2011/160932

[12] Abe, E. and Omo-Aghoja, L.O. (2009) Maternal Mortality at the Central Hospital, Benin City Nigeria: A Ten Year Review.

[13] Lee, Q.Y., Odoi, A.T., Opare-Addo, H. and Dassah, E.T. (2012) Maternal Mortality in Ghana: A Hospital-Based Review. Acta Obstetricia et Gynecologica Scandinavica, 91, 87-92. https://doi.org/10.1111/j.1600-0412.2011.01249.x

[14] Dasari, P. (2015) Maternal Mortality and Its Relationship to Emergency Obstetric Care (EmOC) in a Tertiary Care Hospital in South India. Obstetric Medicine, 8, 86-91. https://doi.org/10.1177/1753495X15575949

[15] Mpemba, F., Kampo, S. and Zhang, X. (2013) Towards 2015: Post-Partum Haemorrhage in Sub-Saharan Africa Still on the Rise. Journal of Clinical Nursing, 23, 774-783. https://doi.org/10.1111/jocn.12126

[16] Ezugwu, E.C., Agu, P.U., Nwoke, M.O. and Ezugwu, F.O. (2014) Reducing Maternal Deaths in a Low Resource Setting in Nigeria. Nigerian Journal of Clinical Practice, 17, 62-66. https://doi.org/10.4103/1119-3077.122842

[17] Shakur, H., Roberts, I., Fawole, B., Chaudhri, R., El-Sheikh, M., Akintan, A., et al. (2017) Effect of Early Tranexamic Acid Administration on Mortality, Hysterectomy, and Other Morbidities in Women with Post-Partum Haemorrhage (WOMAN): An International, Randomised, Double-Blind, Placebo-Controlled Trial. The Lancet, 389, 2105-2116.

[18] Tort, J., Rozenberg, P., Traoré, M., Fournier, P. and Dumont, A. (2015) Factors Associated with Postpartum Hemorrhage Maternal Death in Referral Hospitals in Senegal and Mali: A Cross-Sectional Epidemiological Survey. BMC Pregnancy Childbirth, 15, 235. https://doi.org/10.1186/s12884-015-0669-y 
[19] Prual, A., Bouvier-Colle, M.-H., Bernis, L. and Bréart, G. (2000) Severe Maternal Morbidity from Direct Obstetric Causes in West Africa: Incidence and Case Fatality Rates. Bulletin of the World Health Organization, 78, 593-602.

[20] Kaye, D.K., Kakaire, O. and Osinde, M.O. (2011) Systematic Review of the Magnitude and Case Fatality Ratio for Severe Maternal Morbidity in Sub-Saharan Africa between 1995 and 2010. BMC Pregnancy Childbirth, 11, 65.

https://doi.org/10.1186/1471-2393-11-65

[21] Belinga, E., Foumane, P., Dohbit, S.J., Um, E.M.N., Kiyeck, D.K. and Mboudou, E.T. (2017) Pronostic des références obstétricales à l'Hôpital Gynéco-Obstétrique et Pédiatrique de Yaoundé (HGOPY). Pan African Medical Journal, 28, 301. https://doi.org/10.11604/pamj.2017.28.301.10773

[22] Dassah, E.T., Kusi-Mensah, E., Morhe, E.S.K. and Odoi, A.T. (2019) Maternal and Perinatal Outcomes among Women with Hypertensive Disorders in Pregnancy in Kumasi, Ghana. PLoS ONE, 14, e0223478. https://doi.org/10.1371/journal.pone.0223478

[23] Mooij, R., Lugumila, J., Mwashambwa, M.Y., Mwampagatwa, I.H., van Dillen, J. and Stekelenburg, J. (2015) Characteristics and Outcomes of Patients with Eclampsia and Severe Pre-Eclampsia in a Rural Hospital in Western Tanzania: A Retrospective Medical Record Study. BMC Pregnancy Childbirth, 15, 213.

https://doi.org/10.1186/s12884-015-0649-2

[24] Zwart, J.J., Dupuis, J.R.O., Richters, A., Öry, F. and van Roosmalen, J. (2010) Obstetric Intensive Care Unit Admission: A 2-Year Nationwide Population-Based Cohort Study. Intensive Care Medicine, 36, 256-263. https://doi.org/10.1007/s00134-009-1707-x

[25] Committee on Obstetric Practice (2017) Committee Opinion No. 623: Emergent Therapy for Acute-Onset, Severe Hypertension during Pregnancy and the Postpartum Period. Obstetrics \& Gynecology, 129, e90-e95. https://doi.org/10.1097/AOG.0000000000002019

[26] Oteng-Ntim, E., Ayensah, B., Knight, M. and Howard, J. (2015) Pregnancy Outcome in Patients with Sickle Cell Disease in the UK: A National Cohort Study Comparing Sickle Cell Anaemia (HbSS) with HbSC Disease. British Journal of Haematology, 169, 129-137. https://doi.org/10.1111/bjh.13270

[27] Barfield, W.D., Barradas, D.T., Manning, S.E., Kotelchuck, M. and Shapiro-Mendoza, C.K. (2010) Sickle Cell Disease and Pregnancy Outcomes: Women of African Descent. American Journal of Preventive Medicine, 38, S542-S549. https://doi.org/10.1016/j.amepre.2009.12.020

[28] Al Jama, F.E., Gasem, T., Burshaid, S., Rahman, J., Al Suleiman, S.A. and Rahman, M.S. (2009) Pregnancy Outcome in Patients with Homozygous Sickle Cell Disease in a University Hospital, Eastern Saudi Arabia. Archives of Gynecology and Obstetrics, 280, 793-797. https://doi.org/10.1007/s00404-009-1002-7

[29] Oteng-Ntim, E., Meeks, D., Seed, P.T., Webster, L., Howard, J., Doyle, P., et al. (2015) Adverse Maternal and Perinatal Outcomes in Pregnant Women with Sickle Cell Disease: Systematic Review and Meta-Analysis. Blood, 125, 3316-3325. https://doi.org/10.1182/blood-2014-11-607317

[30] Muganyizi, P.S. and Kidanto, H. (2013) Sickle Cell Disease in Pregnancy: Trend and Pregnancy Outcomes at a Tertiary Hospital in Tanzania. PLoS ONE, 8, e56541. https://doi.org/10.1371/journal.pone.0056541 


\section{Appendix}

Determinants of post operative infection following gynecological and obstetrical surgeries in the Douala Gynaeco-obstetric and Paediatric Hospital.

\section{Data Collection Sheet}

\section{1) Identification}

a) Name ID: DATE:

b) Age (years):

d) Residence:

e) Level of education:
i) Primary
ii) Secondary
iii) University

2) Past history

a) Chronic disease:
i) Sickle cell anaemia
ii) Diabetes
iii) HTN (hypertension)
iv) Cancer
v) other

b) Past surgery:
i) $\mathrm{No}$
ii) Yes
Indication

c) Tobacco consumption:

i) No

ii) Yes

d) Chronic infection:
i) HIV
ii) Tuberculosis
iii) Hepatitis

3) Patient's condition at the time of diagnosis of $\mathrm{OE}$

a) Primary site of management:
i) $\mathrm{DGOPH}$
ii) other hospital

b) Timing of Management: time of arrival time management started

c) Type of OE:
i) $\mathrm{EP}$
ii) HDP
iii) $\mathrm{PPH}$
iv) $A B$
v) $\mathrm{OL}$
vi) SCA
vii) $\mathrm{PP}$
viii) $A P$
ix) AFD,
x) Malaria
xi) HIV-AIDS
xii) Cardiac disease
xiii) Other

d) Health carers present:
i) Emergency nurse
ii) emergency physician
iii) obstetrician
iv) Anaesthetist
v) midwive
vi) other

4) Management and maternal outcome
a) Patient management according to protocol:
i) Yes
ii) No
b) Patient needed ICU:
i) Yes
ii) No
c) Patient admitted in ICU:
i) Yes
ii) No
d) Duration of hospital stay
e) Treatment outcome:
i) Total recovery
ii) referred to other hospital
iii) death

5) Causes of death
a) NA
b) Late referral
c) Critical condition of patient 
d) Inappropriate care

\section{Liste of Abbreviations}

AB: abortions

AFD: acute foetal distress

AP: abruption placentae

EP: ectopic pregnancy

HDP: hypertensive diseases in pregnancy

ICU: intensive care unit

MMR: maternal mortality rate

MIS: management in the site

PEE: preeclampsia/eclampsia

PP: placenta previa

PPH: post partum haemorrhage

OE: obstetric emergencies

OIC: other indirect causes

OL: obstructed labour

SCA: sickle cell anaemia 